\title{
FIXED-POINT FREE MAPS OF EUCLIDEAN SPACES
}

\author{
R. Z. BUZYAKOVA AND A. CHIGOGIDZE
}

\begin{abstract}
Our main result states that every fixed-point free continuous self-map of $\mathbb{R}^{n}$ is colorable. This result can be re-formulated as follows: A continuous map $f: \mathbb{R}^{n} \rightarrow \mathbb{R}^{n}$ is fixed-point free iff $\widetilde{f}: \beta \mathbb{R}^{n} \rightarrow \beta \mathbb{R}^{n}$ is fixedpoint free. We also obtain a generalization of this fact and present some examples.
\end{abstract}

\section{INTRODUCTION}

It is known that for a continuous map $f: X \rightarrow X$ the set of all fixed points $\operatorname{Fix}(\widetilde{f})$ of its Stone-Čech extension $\widetilde{f}: \beta X \rightarrow \beta X$ may differ from $\operatorname{cl}_{\beta X} \operatorname{Fix}(f)$. In particular, $\operatorname{Fix}(\widetilde{f})$ could be non-empty for a fixed-point free $f$, which is equivalent to non-colorability of $f$ if $X$ is normal. Since the just used notion of colorability may not be widely known let us define it.

Definition of Colorability. $f: X \subset Y \rightarrow Y$ is colorable if one can cover $X$ by finitely many closed sets each of which misses its image under $f$.

Metric examples (constructed by Krzysztof Mazur and van Douwen) of fixedpoint free non-colorable maps can be found in [5], 3], 6], 8]. It is interesting to note that these examples are either infinite-dimensional or non-locally compact. On the other hand, it is known that: (a) every fixed-point free autohomeomorphism $f$ of a finite-dimensional paracompact space $X$ has the fixed-point free extension $\tilde{f}$ (see [3]), and (b) every continuous fixed-point free map $f$ of a zero-dimensional sigma-compact space $X$ has the fixed-point free extension $\widetilde{f}$ (see [6]). We also mention here recently obtained result [1] stating colorability of every continuous fixed-point free self-map of the real line $\mathbb{R}$.

Below we show (Theorem 2.6) that every continuous fixed-point free self-map of the Euclidean space $\mathbb{R}^{n}$ is colorable. In fact, we show more by considering not only self-maps $f: X \rightarrow X$, but also maps $f: X \rightarrow Y$, defined on closed subspaces $X$ of $Y$. This version shows itself helpful in various situations and in our opinion is of interest. The most general statement we present in this paper is Theorem 3.6 which states that every continuous fixed-point free map $f: X \rightarrow Y$, defined on a closed subspace $X$ of an at most $n$-dimensional locally

1991 Mathematics Subject Classification. 54H25, 58C30.

Key words and phrases. fixed-point free map, colorable map. 
compact and paracompact space $Y$, is colorable in at most $n+3$ colors. We also note that a continuous map $f: X \rightarrow Y$, defined on a closed subspace $X$ of an at most $n$-dimensional locally compact and paracompact space $Y$, is fixed-point free iff $\widetilde{f}: \beta X \rightarrow \beta Y$ is fixed-point free. In Section 4, we construct a noncolorable fixed-point free autohomeomorphism of the separable Hilbert space $\ell_{2}$ and a non-colorable continuous fixed-point free self-map of the universal $n$ dimensional Nöbeling space $N_{n}^{2 n+1}$. The case $n=0$ produces (and uses) the same example as that of K. Mazur's since $N_{0}^{1}$ is the space of irrationals. We conclude by proving (Proposition 4.5) that similar examples exist on every zerodimensional non-sigma-compact Polish space.

A good account of the results related to this subject as well as historical comments are given in [8, Section 3.2]. Our notation and terminology related to absolute extensors in dimension $n, n$-soft maps, universal Nöbeling spaces and inverse spectra follow [2].

When discussing the $n$-th power $\mathbb{R}^{n}$ of $\mathbb{R}$ we agree that $\mathbb{R}^{0}$ is the singleton $\{0\}$. We finish the introduction by defining colorability-related concepts used in the paper. Once again, a continuous map $f: X \rightarrow Y$ is colorable if there exists a finite cover $\mathcal{F}$ of $X$ by its closed subsets such that $f(F)$ misses $F$ for every $F \in \mathcal{F}$. If $\mathcal{F}$ has $n$ elements we say that $f$ is colorable in $n$ colors. Every closed set $F \subset X$ with the property that $f(F) \cap F=\emptyset$ is a color (for $f$ ). All maps under discussion are continuous.

\section{Fixed-POINT FREE MAPS OF EUCLIDEAN SPACES}

The following lemma consists of common facts about colorable maps that are proved implicitly for self-maps in other papers on the subject. Since the proof is a straightforward verification we will only sketch it.

Lemma 2.1. Let $X$ be a closed subspace of $Z$ and $f: X \rightarrow Z$ a continuous map.

(1) If $Z$ is normal, $A$ is a color, and $A$ misses $\overline{f(A)}$; then there is an open neighborhood $U$ of $A$ whose closure is a color.

(2) If $A \subset Z$ and $f(A) \cap A=\emptyset$ then $f^{-1}(A) \cap A=\emptyset$.

(3) If $\mathcal{G}$ is a closed cover of $Z$ such that $f(G) \cap G=\emptyset$ then $\mathcal{F}=\left\{f^{-1}(G)\right.$ : $G \in \mathcal{G}\}$ is a cover of $X$ by colors and $\overline{f(F)} \cap F=\emptyset$ for all $F \in \mathcal{F}$.

Proof. To prove 2, assume the contrary and pick $x$ in $f^{-1}(A) \cap A=\emptyset$. Since $f^{-1}(A)$ is a subset of $X$, the value $f(x)$ is defined. Hence $f \circ f^{-1}(A)$ meets $f(A)$, contradicting $f(A) \cap A=\emptyset$.

To prove 3, apply 2 to conclude that $f^{-1}(G)$ is a color. Since $\mathcal{G}$ is a cover of $Z$ and $f$ is defined on $X$ only, $\mathcal{F}$ is a cover of $X$. Finally, let us show that $\overline{f(F)} \cap F=\emptyset$. We have $F=f^{-1}(G)$ for $G \in \mathcal{G}$. Then $\overline{f(F)} \cap F$ equals 
$\overline{f \circ f^{-1}(G)} \cap f^{-1}(G)$, which is a subset of $\bar{G} \cap f^{-1}(G)$. Since $G$ is closed $\bar{G} \cap$ $f^{-1}(G)$ equals $G \cap f^{-1}(G)$, which is empty by 2 .

We will use the above facts quite often without formally referring to the statement.

Lemma 2.2. Let $X$ be a normal space of dimension at most $n$. Suppose $\left\{A_{i}, B_{i}\right.$ : $i \leq n+1\}$ is a family of functionally closed sets such that $A_{i} \cap B_{i}=\emptyset$. Then there exists a functionally closed cover $\left\{\tilde{A}_{i}, \tilde{B}_{i}: i \leq n+1\right\}$ of $X$ with the following properties:

(1) $\tilde{A}_{i} \cap \tilde{B}_{i}=\emptyset$; and

(2) $\tilde{A}_{i} \cap Z=A_{i}$ and $\tilde{B}_{i} \cap Z=B_{i}$, where $Z=\bigcup\left\{A_{i}, B_{i}: i \leq n+1\right\}$.

Proof. For each $i=1, \ldots, n+1$, consider a map $f_{i}: X \rightarrow I=[0,1]$ such that $f_{i}^{-1}(0)=A_{i}$ and $f_{i}^{-1}(1)=B_{i}$. The diagonal product $f=\triangle f_{i}: X \rightarrow[0,1]^{n+1}$ sends the union $\cup\left(A_{i} \cup B_{i}\right)$ into $\partial I^{n+1}$. Since $\operatorname{dim} X \leq n$ and since $\partial I^{n+1} \simeq S^{n}$ is an absolute extensor in dimension $n$ for normal spaces, it follows that the map $f \mid \cup\left(A_{i} \cup B_{i}\right): \cup\left(A_{i} \cup B_{i}\right) \rightarrow \partial I^{n+1}$ has an extension $g: X \rightarrow \partial I^{n+1}$. The sets $\widetilde{A}_{i}, \widetilde{B}_{i}$ can now be defined as inverse images under the map $g$ of the opposite faces of $\partial I^{n+1}$. The needed properties hold by construction.

To prove our main result for $\mathbb{R}^{n}$ and a generalization to $n$-dimensional locally compact paracompact spaces we encounter several cases that can be handled by one approach realized in Lemma 2.4. To formulate the lemma to a required generality we need the following definition.

Definition of Favorable Representation. A family $\left\{X_{\alpha}: \alpha \leq\right.$ $\tau, \alpha$ is isolated $\}$ is a favorable representation of a space $X$ if all of the following hold:

(1) $X=\bigcup_{\alpha<\tau} X_{\alpha}$;

(2) If $A \cap X_{\alpha}$ is functionally closed in $X_{\alpha}$ for all isolated $\alpha \leq \tau$, then $A$ is functionally closed in $X$;

(3) If $X_{\alpha} \cap X_{\beta} \neq \emptyset$, then $\alpha=\beta+1$ or $\beta=\alpha+1$;

(4) The union of any subcollection of $\left\{X_{\alpha}: \alpha \leq \tau, \alpha\right.$ is isolated $\}$ is functionally closed in $X$.

A much simpler version of Lemma 2.4 for the real line is proved in [1, Lemma 2.1]. To prove our generalized version we introduce three technical definitions that will be used only to prove Lemma 2.4. In what follows when dealing with a favorable representation $\left\{X_{\alpha}: \alpha \leq \tau, \alpha\right.$ is isolated $\}$ we always assume that $X_{\alpha}$ is empty if $\alpha \leq \tau$ is limit. 
Definition of Odd Ordinal. An ordinal $\alpha$ will be called odd if $\mid\{\beta<\alpha$ : $|\alpha \backslash \beta|$ is finite $\} \mid$ is an odd number.

Definition of $\beta$-Coloring. Let $\left\{Y_{\alpha}: \alpha \leq \tau, \alpha\right.$ is isolated $\}$ be a favorable representation of $Y$; $\operatorname{dim} Y \leq n$; and $f: X \subset Y \rightarrow Y$. We say that $\left\{A_{i}, B_{i}\right.$ : $i \leq n+1\}$ is a $\beta$-coloring of $f$ if all of the following hold:

$\mathrm{C} 1:\left\{A_{i}, B_{i}: i \leq n+1\right\}$ is a functionally closed cover of $\bigcup_{\gamma \leq \beta} Y_{\gamma}$.

$\mathrm{C} 2: A_{i} \cap B_{i}=\emptyset$;

C3: $f\left(A_{i}\right) \cap A_{i}=\emptyset$ and $f\left(B_{i}\right) \cap B_{i}=\emptyset$.

Definition of Agreed Colorings. Let $\left\{Y_{\alpha}: \alpha \leq \tau, \alpha\right.$ is isolated $\}$ be a favorable representation of $Y$; $\operatorname{dim} Y \leq n ; f: X \subset Y \rightarrow Y$; and $\left\{A_{i}^{\gamma}, B_{i}^{\gamma}: i \leq\right.$ $n+1\}$ and $\left\{A_{i}^{\beta}, B_{i}^{\beta}: i \leq n+1\right\}$ are $\gamma$-and $\beta$-coloring of $f$ with $\gamma+2 \leq \beta$. We say that the colorings agree if all of the following hold:

A1: If $\gamma$ and $\beta$ are odd then $A_{i}^{\gamma} \subset A_{i}^{\beta}$ and $B_{i}^{\gamma} \subset B_{i}^{\beta}$;

A2: If $\beta$ is odd and $\gamma^{\prime}$ is the smallest odd ordinal greater than or equal to $\gamma$, then $A_{i}^{\beta} \cap Y_{\gamma}=A_{i}^{\gamma^{\prime}+2} \cap Y_{\gamma}$ and $B_{i}^{\beta} \cap Y_{\gamma}=B_{i}^{\gamma^{\prime}+2} \cap Y_{\gamma}$.

Observe that in A2 of the above definition, $\gamma^{\prime}+2 \leq \beta$ because $\beta$ is odd and $\beta \geq \gamma+2$. What the requirement $\mathrm{A} 2$ states is that $A_{i}^{\beta}$ and $A_{i}^{\alpha}$ cut out the same piece from $Y_{\gamma}$ as long as $\alpha$ and $\beta$ are odd and at least two ordinals above $\gamma$. This property together with 2 of favorable representation will be used below to show that the union of $A_{i}^{\beta}$, s over add $\beta$ is closed.

Lemma 2.3. Let $\left\{Y_{\alpha}: \alpha \leq \tau, \alpha\right.$ is isolated $\}$ be a favorable representation of a normal space $Y$ of dimension at most $n$. Let $X$ be a closed subspace of $Y$ and $f: X \rightarrow Y$ a continuous map. Let $\alpha$ be limit and $\left\{\left\{A_{i}^{\beta}, B_{i}^{\beta}: i \leq n+1\right\}\right.$ : $\beta<\alpha\}$ be a collection of $\beta$-colorings of $f$ that agree with each other. Then the following sets form an $\alpha$-coloring of $f$ that agrees with all $\beta$-colorings in the given collection:

$A_{i}^{\alpha}=\bigcup\left\{B_{i}^{\beta}: \beta<\alpha, \beta\right.$ is odd $\}, B_{i}^{\alpha}=\bigcup\left\{A_{i}^{\beta}: \beta<\alpha, \beta\right.$ is odd $\}$, where $i \leq n+1$.

Proof. Neither A1 nor A2 in the definition of agreed colorings needs verification since being limit $\alpha$ is not odd. Let us verify C1-C3 in the definitions of $\alpha$ coloring. For $\mathrm{C} 1$, recall that $Y_{\alpha}=\emptyset$. Therefore, if $x \in \bigcup_{\beta \leq \alpha} Y_{\beta}$ then $x \in Y_{\beta}$ for $\beta<\alpha$. Let $\gamma<\alpha$ be an odd ordinal greater than $\beta$. Then by $\mathrm{C} 1$ for $\gamma$, we have $x \in \bigcup\left\{A_{i}^{\gamma}, B_{i}^{\gamma}: i \leq n+1\right\}$. Thus, $\left\{A_{i}^{\alpha}, B_{i}^{\alpha}: i \leq n+1\right\}$ is a cover of $\bigcup_{\beta \leq \alpha} Y_{\beta}$. Let us show that $A_{i}^{\alpha}$ is functionally closed in $Y$. By property 2 of favorable representations we need to fix an isolated $\gamma$ and show that $Y_{\gamma} \cap A_{i}^{\alpha}$ is functionally closed in $Y_{\gamma}$. The inclusion $A_{i}^{\alpha} \subset \bigcup_{\beta \leq \alpha} Y_{\beta}$, the equality $Y_{\alpha}=\emptyset$, and 3 of favorable representation imply that $A_{i}^{\alpha}$ may meet only $Y_{\gamma}$ with $\gamma<\alpha$. 
We have $A_{i}^{\alpha} \cap Y_{\gamma}=\bigcup\left\{B_{i}^{\beta} \cap Y_{\gamma}: \beta<\alpha, \beta\right.$ is odd $\}$. This set can be written as the union of two sets $T_{1}=\bigcup\left\{B_{i}^{\beta} \cap Y_{\gamma}: \gamma^{\prime}+2 \leq \beta<\alpha, \beta\right.$ is odd $\}$ and $T_{2}=\bigcup\left\{B_{i}^{\beta} \cap Y_{\gamma}: \beta \leq \gamma^{\prime}+2, \beta\right.$ is odd $\}$, where $\gamma^{\prime}$ is the smallest odd ordinal greater than or equal to $\gamma$. The set $T_{1}$ is $B_{i}^{\gamma^{\prime}+2} \cap Y_{\gamma}$ by A2 for ordinals below $\alpha$. The set $B_{i}^{\gamma^{\prime}+2}$ is functionally closed by inductive assumption C1. Therefore, $B_{i}^{\gamma^{\prime}+2} \cap Y_{\gamma}$ is functionally closed in $Y_{\gamma}$. The set $T_{2}$ is $B_{i}^{\gamma^{\prime}+2} \cap Y_{\gamma}$ by A1 for ordinals below $\alpha$ and is functionally closed for the same reasons as the first one. Thus, $A_{i}^{\alpha} \cap Y_{\gamma}$ is the union of two functionally closed sets in $Y_{\gamma}$.

For C2, we need to show that $A_{i}^{\beta} \cap B_{i}^{\gamma}=\emptyset$ for odd $\beta, \gamma<\alpha$. Assume $\gamma \geq \beta$. By $\mathrm{A} 1$ for $\gamma$, we have $A_{i}^{\beta} \subset A_{i}^{\gamma}$. By C2 for $\gamma$, we have $A_{i}^{\gamma} \cap B_{i}^{\gamma}=\emptyset$.

For C3, we need to show that $f\left(B_{i}^{\beta}\right) \cap B_{i}^{\gamma}=\emptyset$ for odd $\beta, \gamma<\alpha$. Assume $\gamma \geq \beta$. By A1 for $\gamma$, we have $B_{i}^{\beta} \subset B_{i}^{\gamma}$. By C3 for $\gamma$, we have $f\left(B_{i}^{\gamma}\right) \cap B_{i}^{\gamma}=\emptyset$.

Lemma 2.4. Let $\left\{Y_{\alpha}: \alpha \leq \tau, \alpha\right.$ is isolated $\}$ be a favorable representation of a normal space $Y$ of dimension at most $n$. Let $X$ be a closed subspace of $Y$ and $f: X \rightarrow Y$ a fixed-point free continuous map with the following properties:

(1) $X$ misses $Y_{0}$; and

(2) If $z \in Y_{\alpha} \cap X$ then $f(z) \in\left[\bigcup_{\beta<\alpha} Y_{\beta}\right] \backslash Y_{\alpha}$.

Then there exists a $(2 n+2)$-sized coloring $\mathcal{F}$ of $f$ such that $c_{Y}(f(F)) \cap F=\emptyset$ for every $F \in \mathcal{F}$.

Proof. Inductively, we will construct building blocks for our colors.

Step 0: Put $A_{1}^{0}=Y_{0}, A_{i}^{0}=\emptyset$ if $1<i \leq n+1$, and $B_{i}^{0}=\emptyset$ if $i \leq n+1$.

Induction Assumption: Assume that for $\beta<\alpha$ we have defined families

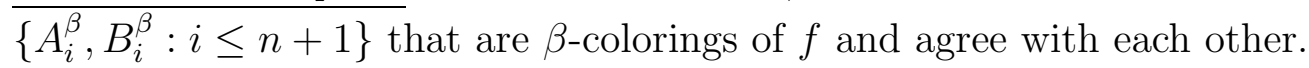

Observe that for $\beta=0$ the family $\left\{A_{i}^{0}, B_{i}^{0}: i \leq n+1\right\}$ meets $\mathrm{C} 1-\mathrm{C} 3, \mathrm{~A} 1$, and A2 in the definitions of $\beta$-coloring and agreed colorings. Indeed, $\mathrm{C} 1$ holds because $A_{1}^{0}=Y_{0}$. C2 holds because $B_{i}^{0}=\emptyset$. C3 holds because $f$ is not defined on $Y_{0}$. A1 and A2 are not applicable for $\beta=0$ since 0 is not odd.

$\underline{\text { Step }[\alpha=\lim \{\beta<\alpha\}]}$ : We construct $\alpha$-coloring as in Lemma 2.3 .

Step $[\alpha=\beta+1]:$ Put

$$
C_{i}^{\alpha}=\left[f^{-1}\left(A_{i}^{\beta}\right) \cap Y_{\alpha}\right] \cup B_{i}^{\beta} \text { and } D_{i}^{\alpha}=\left[f^{-1}\left(B_{i}^{\beta}\right) \cap Y_{\alpha}\right] \cup A_{i}^{\beta} .
$$

Let us show that these sets have the following properties:

S1. $C_{i}^{\alpha}$ and $D_{i}^{\alpha}$ are functionally closed;

S2. $C_{i}^{\alpha} \cap D_{i}^{\alpha}=\emptyset$; and 
S3. If $x \in \bigcup_{\gamma \leq \alpha} Y_{\gamma} \cap X$ then both $f(x)$ and $x$ are in $\bigcup\left\{C_{i}^{\alpha}, D_{i}^{\alpha}: i \leq n+1\right\}$. For $\mathrm{S} 1$, observe that $f^{-1}\left(A_{i}^{\beta}\right)$ is functionally closed as the inverse image of the set functionally closed by $\mathrm{C} 1$ for $\beta$. By property 4 of favorable representation, $f^{-1}\left(A_{i}^{\beta}\right) \cap Y_{\alpha}$ is functionally closed in $Y$ as well. The set $B_{i}^{\beta}$ is functionally closed by $\mathrm{C} 1$ for $\beta$. Thus, $C_{i}^{\alpha}$ is functionally closed. For $\mathrm{S} 2$ we need to verify the following four equalities:

(1) $\left[f^{-1}\left(A_{i}^{\beta}\right) \cap Y_{\alpha}\right] \cap\left[f^{-1}\left(B_{i}^{\beta}\right) \cap Y_{\alpha}\right]=\emptyset$;

(2) $\left[f^{-1}\left(A_{i}^{\beta}\right) \cap Y_{\alpha}\right] \cap A_{i}^{\beta}=\emptyset$;

(3) $B_{i}^{\beta} \cap\left[f^{-1}\left(B_{i}^{\beta}\right) \cap Y_{\alpha}\right]=\emptyset$; and

(4) $B_{i}^{\beta} \cap A_{i}^{\beta}=\emptyset$.

Equality 1 holds because $A_{i}^{\beta} \cap B_{i}^{\beta}=\emptyset$ by $\mathrm{C} 2$ for $\beta$. Equalities 2 and 3 hold by $\mathrm{C} 3$ for $\beta$. Equality 4 holds by $\mathrm{C} 2$ for $\beta$. Let us show $\mathrm{S} 3$. By 2 of the lemma hypothesis, $f(x) \in \bigcup_{\gamma \leq \beta} Y_{\gamma}$ and $x \in\left[f^{-1}\left(\bigcup_{\gamma \leq \beta} Y_{\gamma}\right) \cap Y_{\alpha}\right] \cup\left[\bigcup_{\gamma \leq \beta} Y_{\gamma}\right]$. By $\mathrm{C} 1$ for $\beta, \bigcup_{\gamma \leq \beta} Y_{\gamma}=\bigcup\left\{A_{i}^{\beta}, B_{i}^{\beta}: i \leq n+1\right\}$ and $\left[f^{-1}\left(\bigcup_{\gamma \leq \beta} Y_{\gamma}\right) \cap Y_{\alpha}\right] \subset$ $\bigcup\left\{f^{-1}\left(A_{i}^{\beta}\right) \cap Y_{\alpha}, f^{-1}\left(B_{i}^{\beta}\right) \cap Y_{\alpha}: i \leq n+1\right\}$. The right-hand sets in these formulas are subsets of $\bigcup\left\{C_{i}^{\alpha}, D_{i}^{\alpha}: i \leq n+1\right\}$ by the definition.

Since $C_{i}^{\alpha}$ and $D_{i}^{\alpha}$ are functionally closed and disjoint, by Lemma 2.2, there exists a family $\left\{A_{i}^{\alpha}, B_{i}^{\alpha}: i \leq n+1\right\}$ of subsets of $\bigcup_{\gamma \leq \alpha} Y_{\gamma}$ with the following properties:

S4. $\left\{A_{i}^{\alpha}, B_{i}^{\alpha}: i \leq n+1\right\}$ is a functionally closed cover of $\bigcup_{\gamma \leq \alpha} Y_{\gamma}$;

S5. $A_{i}^{\alpha} \cap Z=C_{i}^{\alpha}$ and $B_{i}^{\alpha} \cap Z=D_{i}^{\alpha}$, where $Z=\bigcup\left\{C_{j}^{\alpha}, D_{j}^{\alpha}: j \leq n+1\right\}$;

S6. $A_{i}^{\alpha} \cap B_{i}^{\alpha}=\emptyset$.

In addition, if $x \in A_{i}^{\alpha} \cap X$ then, by S3, $x \in Z=\bigcup\left\{C_{i}^{\alpha}, D_{i}^{\alpha}: i \leq n+1\right\}$. Thus, $x \in A_{i}^{\alpha} \cap Z$. By S5, $x \in C_{i}^{\alpha}$. In short,

S7. If $x \in A_{i}^{\alpha} \cap X\left(B_{i}^{\alpha} \cap X\right)$, then $x \in C_{i}^{\alpha}\left(D_{i}^{\alpha}\right)$.

Also, if $x \in A_{i}^{\alpha} \backslash C_{i}^{\alpha}$ then, by S5, $x \notin Z$. The set $Z$ contains $\bigcup_{\gamma \leq \beta} Y_{\gamma}$ due to second summands in the definitions of $C_{i}^{\alpha}$ and $D_{i}^{\alpha}$ and assumption C1 for $\beta$. Therefore, $x \notin \bigcup_{\gamma \leq \beta} Y_{\gamma}$. Hence, $x \in Y_{\alpha}$. In summary,

S8. If $x \in A_{i}^{\alpha} \backslash C_{i}^{\alpha}$ then $x \in Y_{\alpha}$.

Let us check C1-C3, A1, and A2. Property $\mathrm{C} 1$ is $\mathrm{S} 4$ and $\mathrm{C} 2$ is $\mathrm{S} 6$. Let us demonstrate C3 for $A_{i}^{\alpha}$. By S7, $f\left(A_{i}^{\alpha}\right) \cap A_{i}^{\alpha}=f\left(C_{i}^{\alpha}\right) \cap A_{i}^{\alpha}$. By S3, $f\left(C_{i}^{\alpha}\right) \subset$ $Z=\bigcup\left\{C_{i}^{\alpha}, D_{i}^{\alpha}: i \leq n+1\right\}$. By S5, $A_{i}^{\alpha} \cap Z=C_{i}^{\alpha}$. Therefore $f\left(C_{i}^{\alpha}\right) \cap A_{i}^{\alpha}=$ $f\left(C_{i}^{\alpha}\right) \cap C_{i}^{\alpha}$. To show that the last intersection is empty we need to verify the following equalities.

(1) $f\left(f^{-1}\left(A_{i}^{\beta}\right) \cap Y_{\alpha}\right) \cap\left[f^{-1}\left(A_{i}^{\beta}\right) \cap Y_{\alpha}\right]=\emptyset$;

(2) $f\left(f^{-1}\left(A_{i}^{\beta}\right) \cap Y_{\alpha}\right) \cap B_{i}^{\beta}=\emptyset$;

(3) $f\left(B_{i}^{\beta}\right) \cap\left[f^{-1}\left(A_{i}^{\beta}\right) \cap Y_{\alpha}\right]=\emptyset$; and

(4) $f\left(B_{i}^{\beta}\right) \cap B_{i}^{\beta}=\emptyset$. 
Equality 1 holds by $\mathrm{C} 3$ for $\beta$. Equality 2 holds by $\mathrm{C} 2$ for $\beta$. Let us show 3 . If $\beta=0$ then, by 1 of the lemma's hypothesis, $f\left(B_{i}^{\beta}\right)$ is empty and 3 holds. Now assume $\beta>0$. From 2 of the lemma's hypothesis and emptiness of $Y_{\lambda}$ for limit $\lambda$, we conclude that the first set is in $\bigcup_{\gamma<\beta} Y_{\gamma}$. The second set is in $Y_{\alpha}$ by the definition. By 3 of favorable representation, $\bigcup_{\gamma<\beta} Y_{\gamma}$ misses $Y_{\alpha}$. Hence the intersection is empty. Equality 4 is $\mathrm{C} 3$ for $\beta$.

To verify A1 we assume $\alpha$ is odd and pick an odd $\gamma<\alpha$. By construction, $A_{i}^{\alpha}$ contains $\left[f^{-1}\left(A_{i}^{\beta}\right) \cap Y_{\alpha}\right] \cup B_{i}^{\beta}$. It suffices to show that $B_{i}^{\beta}$ contains $A_{i}^{\gamma}$. If $\beta$ is limit then, by construction, $B_{i}^{\beta}=\bigcup\left\{A_{i}^{\lambda}: \lambda<\beta, \lambda\right.$ is odd $\}$ and the right side contains $A_{i}^{\gamma}$. If $\beta=\lambda+1$ then, by construction, $B_{i}^{\beta}$ contains $\left[f^{-1}\left(B_{i}^{\lambda}\right) \cap Y_{\beta}\right] \cup A_{i}^{\lambda}$. By A1 for $\lambda, A_{i}^{\lambda}$ contains $A_{i}^{\gamma}$.

Finally to show $A 2$ we assume $\alpha$ is odd and pick a $\gamma$ such that $\gamma^{\prime}+2<\alpha$, where $\gamma^{\prime}$ is the smallest odd ordinal greater than or equal to $\gamma$. Observe that $A_{i}^{\alpha}=C_{i}^{\alpha} \cup\left(A_{i}^{\alpha} \backslash C_{i}^{\alpha}\right)$ which in its turn equals $\left[f^{-1}\left(A_{i}^{\beta}\right) \cap Y_{\alpha}\right] \cup B_{i}^{\beta} \cup\left(A_{i}^{\alpha} \backslash C_{i}^{\alpha}\right)$. The first set in this union is in $Y_{\alpha}$ and so is the third by S8. By 3 of favorable representation $Y_{\alpha}$ misses $Y_{\gamma}$. Thus, $A_{i}^{\alpha} \cap Y_{\gamma}=B_{i}^{\beta} \cap Y_{\gamma}$.

If $\beta$ is limit, then $B_{i}^{\beta} \cap Y_{\gamma}=\bigcup\left\{A_{i}^{\lambda} \cap Y_{\gamma}: \lambda<\beta, \lambda i\right.$ s odd $\}$. The right set can be written as the union of $T_{1}=\bigcup\left\{A_{i}^{\lambda} \cap Y_{\gamma}: \lambda \leq \gamma^{\prime}+2, \lambda\right.$ is odd $\}$ and $T_{2}=\bigcup\left\{A_{i}^{\lambda} \cap Y_{\gamma}: \gamma^{\prime}+2 \leq \lambda<\beta, \lambda\right.$ is odd $\}$. By A1, $T_{1}=A_{i}^{\gamma^{\prime}+2} \cap Y_{\gamma}$. By A2, $T_{2}=A_{i}^{\gamma^{\prime}+2} \cap Y_{\gamma}$.

Now assume $\beta$ is isolated. Since there exists odd $\gamma^{\prime}$ below odd $\alpha$ we conclude that $\beta \neq 0$. Therefore, $\beta=\lambda+1$. We have $B_{i}^{\beta}=D_{i}^{\beta} \cup\left(B_{i}^{\beta} \backslash D_{i}^{\beta}\right)$, which in its turn equals the union of three sets $T_{1}=\left[f^{-1}\left(B_{i}^{\lambda}\right) \cap Y_{\beta}\right], T_{2}=A_{i}^{\lambda}$, and $T_{3}=\left(B_{i}^{\beta} \backslash D_{i}^{\beta}\right)$. Since $\alpha>\gamma+2$, we conclude $\beta>\gamma+1$. By property 3 of favorable representations, $Y_{\beta}$ misses $Y_{\gamma}$. We have $T_{1}$ is in $Y_{\beta}$ and so is $T_{3}$ by $\mathrm{S} 8$ for isolated $\beta$. Therefore, only $T_{2}$ can meet $Y_{\gamma}$. Hence, $B_{i}^{\beta} \cap Y_{\gamma}=A_{i}^{\lambda} \cap Y_{\gamma}$. By A2 for $\lambda$, the right side of the last equality is $A_{i}^{\gamma^{\prime}+2} \cap Y_{\gamma}$. Inductive construction is complete.

Put $A_{i}=A_{i}^{\tau}$ and $B_{i}=B_{i}^{\tau}$. By C1-C3, the family $\mathcal{G}=\left\{A_{i}, B_{i}: i \leq n+1\right\}$ is a closed cover of $Y$ such that $f(G) \cap G=\emptyset$ for every $G \in \mathcal{G}$. By 3 of Lemma 2.1, $\mathcal{F}=\left\{f^{-1}(G): G \in \mathcal{G}\right\}$ is a desired coloring of $f$. The lemma is proved.

Lemma 2.5. Let $X$ be a compact subspace of $\mathbb{R}^{n}$ and $f: X \rightarrow \mathbb{R}^{n}$ a continuous fixed-point free map. Then $f$ is colorable in at most $4 n(n+1)$ colors.

Proof. For each $i \leq n$, put $A_{i}=\left\{x \in X: \pi_{i} \circ f(x)>\pi_{i}(x)\right\}$ and $B_{i}=\{x \in$ $\left.X: \pi_{i} \circ f(x)<\pi_{i}(x)\right\}$. By continuity of $f$, each of these sets is open. Since $f$ is fixed-point free, $\left\{A_{i}, B_{i}: i \leq n\right\}$ is a cover of $X$. By paracompactness of $X$, there exists a closed shrinking $\left\{A_{i}^{\prime}, B_{i}^{\prime}: i \leq n\right\}$. It suffices to show now that $\left.f\right|_{S}$ 
is colorable in $2(n+1)$ colors for each $S \in\left\{A_{i}^{\prime}, B_{i}^{\prime}: i \leq n\right\}$. We will demonstrate it for $A=A_{1}^{\prime}$.

Fix $a \in A$. Since $A$ is compact and $\pi_{1} \circ f(x)>\pi_{1}(x)$ for every $x \in A$ we can find $\epsilon_{a}>0$ such that

$(*)$ If $x \in A$ and $\pi_{1}(x) \in\left[\pi_{1}(a)-\epsilon_{a}, \pi_{1}(a)+\epsilon_{a}\right]$ then $\pi_{1} \circ f(x)>\pi_{1}(a)+\epsilon_{a}$.

By compactness and $(*)$ we can select a strictly decreasing sequence of reals $r_{1}>\ldots .>r_{m}$ such that

P1. $A \subset\left[r_{m}, r_{1}\right) \times \mathbb{R}^{n-1}$; and

P2. If $x \in\left[r_{k+1}, r_{k}\right] \times \mathbb{R}^{n-1} \cap A$ then $\pi_{1} \circ f(x)>r_{k}$.

Put $X_{0}=\left[r_{1}, \infty\right) \times \mathbb{R}^{n-1} ; X_{k}=\left[r_{k+1}, r_{k}\right] \times R^{n-1}$ if $1 \leq k \leq m-1$; and $X_{m}=\left(-\infty, r_{m}\right] \times \mathbb{R}^{n-1}$. Clearly $\left\{X_{k}\right\}_{k \leq m}$ is a favorable representation of $\mathbb{R}^{n}$. We only need to show that $\mathbb{R}^{n},\left\{X_{k}\right\}_{k \leq m},\left.f\right|_{A}$, and $A$ meet 1 and 2 of Lemma 2.4. Requirement 1 of Lemma 2.4 is met because $X_{0}$ misses $A$ by P1. Requirement 2 of Lemma 2.4 is met in P2. The lemma is proved.

Theorem 2.6. Let $X$ be a closed subspace of $\mathbb{R}^{n}$ and $f: X \rightarrow \mathbb{R}^{n}$ a continuous fixed-point free map. Then $f$ is colorable.

Proof. Put $X_{1}=[-1,1]^{n}$ and $X_{k+1}=[-(k+1),(k+1)]^{n} \backslash(-k, k)^{n}$. Let $M=4 n(n+1)$.

Claim 1. Let $A=\left\{x \in X: x \in X_{k}\right.$ and $f(x) \in X_{m}$, where $\left.m \geq k\right\}$. Then there exists a finite open cover $\mathcal{U}_{A}$ of $A$ such that the closure of every element of $\mathcal{U}_{A}$ is a color.

Proof of Claim 1. Clearly, $A$ is closed. Fix a strictly increasing sequence $\left\langle b_{k}\right\rangle_{k}$ of positive integers that have the following property.

P1. $f\left(\left[-b_{k}, b_{k}\right]^{n} \cap X\right) \subset\left(-b_{k+1}, b_{k+1}\right)^{n}$.

For every $k$, select $\mathcal{S}_{k}=\left\{S_{1}^{k}, \ldots, S_{M}^{k}\right\}$ a coloring of $f_{k}$, where $f_{k}$ is the restriction of $f$ to $A \cap\left(\left[-b_{k}, b_{k}\right]^{n} \backslash\left(-b_{k-1}, b_{k-1}\right)^{n}\right)$. This is possible by Lemma 2.5. By the definition of $A$, we have $f(S)$ does not meet $\left(-b_{k-1}, b_{k-1}\right)^{n}$ for any $S \in \mathcal{S}_{k}$. Also $f(S)$ does not meet the compliment of $\left(b_{k+1}, b_{k+1}\right)^{n}$ by P1. In short, we have

P2. $f(S) \subset\left(-b_{k+1}, b_{k+1}\right)^{n} \backslash\left(-b_{k-1}, b_{k-1}\right)^{n}$ for every $S \in \mathcal{S}_{k}$.

Put $O_{i}=\bigcup\left\{S_{i}^{k}: k i s\right.$ odd $\}$ and $E_{i}=\bigcup\left\{S_{i}^{k}: k\right.$ is even $\}$. The sets $O_{i}$ and $E_{i}$ are closed as unions of discrete families of closed sets and $\left\{O_{i}, E_{i}: i \leq M\right\}$ is a cover of $A$. Let us show that $f\left(O_{1}\right) \cap O_{1}=\emptyset$. By P2, we have $f\left(S_{1}^{k}\right) \cap S_{1}^{m}=\emptyset$ if $k \neq m$. Since $S_{1}^{k}$ is a color, $f\left(S_{1}^{k}\right) \cap S_{1}^{k}=\emptyset$. Thus we have colored $\left.f\right|_{A}$ in $2 M=8 n(n+1)$ many colors. By 1 of Lemma 2.1, to find a desired open cover it suffices to show that $f\left(O_{i}\right)$ and $f\left(E_{i}\right)$ are closed. We have $f\left(O_{i}\right)=\bigcup\left\{f\left(S_{i}^{k}\right): i\right.$ is odd $\}$. Each 
$f\left(S_{i}^{k}\right)$ is compact since $S_{i}^{k}$ is. By $\mathrm{P} 2,\left\{f\left(S_{i}^{k}\right): i\right.$ is odd $\}$ is a locally finite family. Therefore, $f\left(O_{i}\right)$ is closed. The claim is proved.

Claim 2. Let $B=X \backslash \bigcup \mathcal{U}_{A}$. Then there exists a finite cover $\mathcal{G}_{B}$ of $B$ by colors. Proof of Claim 2. Since $B$ misses $A$ the following holds.

(*) If $x \in B \cap X_{k}$ and $f(x) \in X_{m}$ then $m<k$.

Since $\left\{X_{k}\right\}_{k}$ is a favorable representation of $\mathbb{R}^{n}$ we only need to show that 1 and 2 of Lemma 2.4 are met. Since $X_{1}$ is a subset of $A$ it misses $B$, so 1 of Lemma 2.4 is met. Requirement 2 of Lemma 2.4 is given by $\left(^{*}\right)$. The claim is proved.

Clearly $\mathcal{G}_{B} \cup\left\{\bar{U}: U \in \mathcal{U}_{A}\right\}$ is a finite coloring of $f$.

2.1. Chromatic number. Given a fixed-point free map $f: X \rightarrow Y$, the chromatic number of $f$ is the smallest size of a coloring of $f$. First of all we record the following statement for future references. It extends the main result of [1].

Corollary 2.7. Every continuous fixed-point free self-map of $\mathbb{R}^{n}$ is colorable in at most $n+3$ colors.

Proof. In [8, Theorem 3.12.17] the following theorem (due to R. Pol) is proved: Let $X$ be a separable metrizable space and let $f: X \rightarrow X$ be a fixed-point free continuous map. If $f$ is finitely colorable and $\operatorname{dim} X \leq n$ then $f$ can be colored with $n+3$ colors. This theorem together with Theorem 2.6 give the desired conclusion.

We would like to remark that the theorem cited in the proof of Corollary 2.7 holds if one replace "separable metrizable" by "normal". This fact is observed in [7] and is attributed to Pol. Fur further reference, Theorem 3.12.17 of [8] will be referred to as the Pol theorem.

Next we estimate the chromatic number of continuous fixed-point free maps $f: X \rightarrow \mathbb{R}^{n}$, thus extending Corollary 2.7 and making Theorem 2.6 more precise. For this we need the following observation.

Lemma 2.8. Let $n \geq 0$ and $f: X \rightarrow Y$ be a continuous fixed-point free map defined on a closed subspace $X$ of a metrizable $n$-dimensional AE(n)-space $Y$. Then there exist an embedding $X \subset Y \times[0, \infty)$ and a continuous fixed-point free map $g: Y \times[0, \infty) \rightarrow Y \times[0, \infty)$ such that $g \mid X=f$.

Proof. Let $h: Y \rightarrow Y$ be a continuous extension of $f$. Such $h$ exists since $\operatorname{dim} Y=n$ and $Y \in A E(n)$. Let $\operatorname{Fix}(h)=\{y \in Y: h(y)=y\}$. Since $f$ is fixed-point free and $h \mid X=f$ it follows that $X \cap \operatorname{Fix}(h)=\emptyset$. Identify $Y$ with 
the subspace $Y \times\{0\}$ of the product $Y \times[0, \infty), X$ with the subspace $X \times\{0\}$ and define $g$ as follows:

$$
g(y, r)=(h(y), r+\operatorname{dist}(y, X)) .
$$

For $(x, 0) \in X \times\{0\}$ we have $g(x, 0)=(h(x)$, dist $(x, X))=(f(x), 0)$ which shows that $g$ is an extension of $f$. To see that $g$ is fixed-point free note that

(1) if $y \in \operatorname{Fix}(h)$, then $y \notin X$, and thus $\operatorname{dist}(y, X)>0$ and $g(y, r) \neq(y, r)$;

(2) if $y \notin \operatorname{Fix}(h)$, then $h(y) \neq y$ and thus $g(y, r) \neq(y, r)$.

Proposition 2.9. Every continuous fixed-point free map $f: X \rightarrow Y$, defined on a closed subset $X$ of an at most $n$-dimensional locally compact separable metrizable space $Y$ (e.g. $\left.Y=\mathbb{R}^{n}\right)$, is colorable in at most $n+3$ colors. In addition, there exists an $(n+3)$-sized coloring $\mathcal{F}$ of $f$ such that $F \cap c l_{Y}(f(F))=\emptyset$ for every $F \in \mathcal{F}$.

Proof. Without loss of generality we may assume that $Y$ is a closed subspace of $\mathbb{R}^{2 n+1}$. By Lemma 2.8 and Corollary 2.7, there is a colorable (in at most $2 n+5$ colors) map $g: \mathbb{R}^{2 n+2} \rightarrow \mathbb{R}^{2 n+2}$ such $g \mid X=f$. Let $p: N_{n}^{2 n+1} \rightarrow \mathbb{R}^{2 n+2}$ be an $n$ soft map of the $n$-dimensional universal Nöbelling space (see [2, Theorem 5.6.4]). Since $\operatorname{dim} Y \leq n$ and $p$ is $n$-soft, there exists a map $j: Y \rightarrow N_{n}^{2 n+1}$ such that $p \circ j=\operatorname{id}_{Y}$. Clearly $j(X)$ and $j(Y)$ are copies of $X$ and $Y$ in $N_{n}^{2 n+1}$ and the map $f^{\prime}: j(X) \rightarrow j(Y)$, defined as $f^{\prime}=j \circ f \circ p \mid j(X)$, is a copy of $f$. Consequently, it suffices to prove the required upper bound for the chromatic number of $f^{\prime}$. Using $n$-softness of $p$ we conclude that there exists a map $h: N_{n}^{2 n+1} \rightarrow N_{n}^{2 n+1}$ such that $p \circ h=g \circ p$ and $h|j(X)=j \circ g \circ p| j(X)=j \circ f \circ p \mid j(X)=f^{\prime}$. Colorability of $g$ and the equality $p \circ h=g \circ p$ imply that $h$ is also colorable. Since the dimension of $N_{n}^{2 n+1}$ is $n$, by the Pol theorem [8, Theorem 3.12.17], $h$ is colorable in at most $n+3$ colors. Since $h$ is a self-map, by 3 of Lemma 2.1, we can find $\mathcal{F}$ with the required properties.

\section{Fixed-Point FREe MAPS OF LOCALly COMPACT PARACOMPACT SPACES}

Proposition 3.1. Every continuous fixed-point free map $f: X \rightarrow Y$, defined on a closed subspace of an at most $n$-dimensional locally compact and Lindelöf space $Y$, is colorable in at most $n+3$ colors. In addition, there exists an $(n+3)$-sized coloring $\mathcal{F}$ of $f$ such that $F \cap c l_{Y}(f(F))=\emptyset$ for every $F \in \mathcal{F}$.

Proof. By Proposition 2.9, we may assume that $\omega(Y)>\omega$. First we need the following observation.

Claim. $Y=\lim \mathcal{S}_{Y}$, where $\mathcal{S}_{Y}=\left\{Y_{\alpha}, q_{\alpha}^{\beta}, A\right\}$ is a factorizing $\omega$-spectrum consisting of locally compact separable metrizable spaces $Y_{\alpha}$ and proper projections $p_{\alpha}^{\beta}: Y_{\beta} \rightarrow Y_{\alpha}, \beta \geq \alpha$. 
Proof of Claim. Let $\widetilde{Y}$ be the one-point compactification of $Y$. Represent $\widetilde{Y}$ as the limit of a factorizing $\omega$-spectrum $\mathcal{S}_{\widetilde{Y}}=\left\{\widetilde{Y}_{\alpha}, \widetilde{q}_{\alpha}^{\beta}, B\right\}$, consisting of metrizable compact spaces $\widetilde{Y}_{\alpha}$ and surjective projections. Since $Y$ is locally compact and Lindelöf, it follows that $Y$ is functionally open in $\tilde{Y}$. Since $\mathcal{S}_{\widetilde{Y}}$ is a factorizing spectrum, there exists an index $\alpha_{0} \in B$ such that $Y=\widetilde{q}_{\alpha_{0}}^{-1}\left(\widetilde{q}_{\alpha_{0}}(Y)\right)$. Let $A=$ $\left\{\alpha \in B: \alpha \geq \alpha_{0}\right\}, Y_{\alpha}=\widetilde{q}_{\alpha}(Y)$, and $q_{\alpha}^{\beta}=\widetilde{q}_{\alpha}^{\beta} \mid Y_{\beta}, \beta \geq \alpha, \alpha, \beta \in A$. Then the limit of the spectrum $\mathcal{S}_{Y}=\left\{Y_{\alpha}, q_{\alpha}^{\beta}, A\right\}$ coincides with $Y$. Moreover, each $Y_{\alpha}, \alpha \in A$, is locally compact (as an open subspace of $\widetilde{Y}_{\alpha}$ and each $q_{\alpha}^{\beta}: Y_{\beta} \rightarrow Y_{\alpha}$ is proper (as the restriction of $\widetilde{q}_{\alpha}^{\beta}$ onto the inverse image $Y_{\beta}=\left(\widetilde{q}_{\alpha}^{\beta}\right)^{-1}\left(Y_{\alpha}\right)$ ). Finally, by [2, Corollary 1.3.2], $\mathcal{S}_{Y}$ is a factorizing $\omega$-spectrum. This proves our Claim.

Since $X$ is closed in $Y$ it is the limit of the induced spectrum $\mathcal{S}_{X}=\left\{X_{\alpha}, p_{\alpha}^{\beta}, A\right\}$, where $X_{\alpha}=q_{\alpha}(X)$ and $p_{\alpha}^{\beta}=q_{\alpha}^{\beta} \mid X_{\beta}, \beta \geq \alpha, \alpha, \beta \in A$. Note that $\mathcal{S}_{X}$ is also an $\omega$-spectrum. It is factorizing since so is $\mathcal{S}_{Y}$ and $X$ is $C$-embedded in $Y$.

By [2, Theorems 1.3.4 and 1.3.10], we may assume without loss of generality that each $Y_{\alpha}$ in the spectrum $\mathcal{S}_{Y}$ is at most $n$-dimensional. Again by the Spectral Theorem [2, Theorem 1.3.4], we may assume (if necessary passing to a cofinal and $\omega$-complete subset of $A$ ) that for each $\alpha \in A$ there is a map $f_{\alpha}: X_{\alpha} \rightarrow Y_{\alpha}$ such that $q_{\alpha} \circ f=f_{\alpha} \circ p_{\alpha}$. Since $f$ is fixed-point free and $X$ is Lindelöf, we can find a countable functionally open cover $\left\{G_{i}: i \in \omega\right\}$ of $X$ and a countable collection $\left\{U_{i}: \in \omega\right\}$ of functionally open subsets of $Y$ such that $f\left(G_{i}\right) \subset$ $U_{i}$ and $U_{i} \cap G_{i}=\emptyset$ for each $i \in \omega$. Factorizability of spectra $\mathcal{S}_{X}$ and $\mathcal{S}_{Y}$ guarantees (see [2, Proposition 1.3.1]) the existence of an index $\alpha_{i} \in A$ such that $G_{i}=p_{\alpha_{i}}^{-1}\left(p_{\alpha_{i}}\left(G_{i}\right)\right)$ and $U_{i}=\left(q_{\alpha_{i}}\right)^{-1}\left(q_{\alpha_{i}}\left(U_{i}\right)\right), i \in \omega$. Choose $\beta \in A$ so that $\beta \geq \alpha_{i}$ for each $i \in \omega$ - this is possible because $A$ is an $\omega$-complete set (see [2, Corollary 1.1.28]) - and note that $G_{i}=p_{\beta}^{-1}\left(p_{\beta}\left(G_{i}\right)\right)$ and $U_{i}=$ $\left(q_{\beta}\right)^{-1}\left(q_{\beta}\left(U_{i}\right)\right)$ for each $i \in \omega$. Obviously, $p_{\beta}\left(G_{i}\right) \cap q_{\beta}\left(U_{i}\right)=\emptyset, i \in \omega$. We claim that $f_{\beta}: X_{\beta} \rightarrow Y_{\beta}$ is fixed-point free. Assuming the opposite, pick a point $x \in X_{\beta}$ with $f_{\beta}(x)=x$, choose $y \in X$ so that $p_{\beta}(y)=x$ and pick an index $i \in \omega$ such that $y \in G_{i}=p_{\beta}^{-1}\left(p_{\beta}\left(G_{i}\right)\right)$. Note that $f(y) \in U_{i}$ and $q_{\beta}(f(y)) \in q_{\beta}\left(U_{i}\right)$. But $q_{\beta}(f(y))=f_{\beta}\left(p_{\beta}(y)\right)=f_{\beta}(x)=x \in p_{\beta}\left(G_{i}\right)$ which is impossible since $p_{\beta}\left(G_{i}\right) \cap q_{\beta}\left(U_{i}\right)=\emptyset$.

By Proposition 2.9, $f_{\beta}$ is colorable in $n+3$ colors and there exists a closed cover $\left\{F_{1}, \ldots, F_{n+3}\right\}$ of $X_{\beta}$ such that $c l_{Y_{\beta}}\left(f_{\beta}\left(F_{j}\right)\right) \cap F_{j}=\emptyset$ for each $j=1, \ldots, n+3$. It only remains to note that $\left\{p_{\beta}^{-1}\left(F_{1}\right), \ldots, p_{\beta}^{-1}\left(F_{n+3}\right)\right\}$ is a closed cover of $X$ and $f\left(p_{\beta}^{-1}\left(F_{j}\right)\right) \cap p_{\beta}^{-1}\left(F_{j}\right)=\emptyset$ for each $j=1, \ldots, n+3$.

Lemma 3.2. Let $X$ be a closed subspace of a locally compact paracompact space $Y$ and $f: X \rightarrow Y$ continuous. Then $Y$ can be written as $\oplus\left\{Y_{\alpha}\right.$ : $\alpha$ is isolated, $\alpha \leq \tau\}$, where $Y_{\alpha}$ is Lindelöf and $f\left(X \cap Y_{\alpha}\right) \subset \bigcup_{\beta \leq \alpha} Y_{\beta}$. 
Proof. By Theorem 5.1.27 in [4], $Y$ can be written as $\oplus_{\alpha<\kappa} Z_{\alpha}$, where each $Z_{\alpha}$ is Lindelöf. Suppose for every isolated $\beta<\alpha$, where $\alpha$ is isolated, $Y_{\beta}$ is defined and the following hold:

(1) If $Y_{\beta}$ meets $Z_{\gamma}$ then $Y_{\beta}$ contains $Z_{\gamma}$;

(2) $f\left(Y_{\beta}\right) \subset \bigcup_{\gamma \leq \beta} Y_{\gamma}$;

(3) $Y_{\beta}$ is clopen in $Y$; and

(4) $Y_{\beta}$ is Lindelöf.

If $\bigcup_{\beta<\alpha} Y_{\beta}$ covers all of $Y$ then put $\tau=\alpha$ if $\alpha$ is limit and $\tau=\lambda$ if $\alpha=$ $\lambda+1$ (notice that $\alpha$ cannot be 0 if $Y$ is not empty). The family $\oplus\left\{Y_{\beta}: \beta \leq\right.$ $\tau, \beta$ is isolated $\}$ is a desired sum by properties $1-4$. Otherwise, we define the next piece as follows.

Construction of $Y_{\alpha}$ : Let $\lambda_{0}$ be an ordinal such that $Z_{\lambda_{0}}$ does not meet $\bigcup_{\beta<\alpha} Y_{\beta}$. Put $S_{0}=\left\{\lambda_{0}\right\}$ and, recursively, $S_{n+1}=\left\{\lambda: Z_{\lambda}\right.$ meets $\left.f\left(Z_{\gamma}\right), \gamma \in S_{n}\right\}$. Since $Z_{\gamma}$ is Lindelöf for all $\gamma, f\left(Z_{\gamma}\right)$ can meet only countably many summands in our original free sum. Therefore, $S_{n}$ is countable. Put $Y_{\alpha}=\bigcup\left\{Z_{\gamma}: \gamma \in\right.$ $\bigcup_{n} S_{n}, Z_{\gamma}$ misses $\left.\bigcup_{\beta<\alpha} Y_{\beta}\right\}$. Inductive requirements 1 and 2 are explicitly incorporated in the construction. Requirement 3 is met because $Y_{\alpha}$ is the union of a discrete subfamily of clopen sets. Requirement 4 follows from the Lindelöf property of every $Z_{\gamma}$ and countability of $S_{n}$.

Lemma 3.3. Let $Y=\oplus\left\{Y_{\alpha}: \alpha \leq \tau, \alpha\right.$ is isolated $\}$, where each $Y_{\alpha}$ is locally compact and Lindelöf; $\operatorname{dim} Y \leq n$; and $X$ closed in $Y$. Suppose $f: X \rightarrow Y$ is continuous and the following hold:

(*) $X$ misses $Y_{0}$; and

(**) $f\left(X \cap Y_{\alpha}\right) \subset \bigcup_{\beta<\alpha} Y_{\beta}$ if $\alpha \neq 0$.

Then there exists a coloring $\mathcal{F}$ of $f$ such that $c l_{Y}(f(F)) \cap F=\emptyset$ for every $F \in \mathcal{F}$.

Proof. Clearly, the free sum in the hypothesis is a favorable representation of $Y$. Let us show that the conditions of Lemma 2.4 are met. Requirement 1 of Lemma 2.4 is met by $\left(^{*}\right)$. Requirement 2 of Lemma 2.4 is met by $(* *)$ and the fact that $Y_{\alpha}$ misses $\bigcup_{\beta<\alpha} Y_{\beta}$, which is thanks to our free sum representation.

Theorem 3.4. Any fixed-point free map $f: X \rightarrow Y$, defined on a closed subspace $X$ of a locally compact and paracompact space $Y$ of dimension $\operatorname{dim} Y \leq n$, is colorable. In addition, there exists a coloring $\mathcal{F}$ of $f$ such that $\operatorname{cl}_{Y}(f(F)) \cap F=$ $\emptyset$ for every $F \in \mathcal{F}$. 
Proof. Represent $Y$ as $\oplus\left\{Y_{\alpha}: \alpha \leq \tau, \alpha\right.$ is isolated $\}$ as in the conclusion of Lemma 3.2. For each isolated $\alpha \leq \tau$ not equal 0 , put $Z_{\alpha}=f^{-1}\left(\oplus_{\beta<\alpha} Y_{\beta}\right) \cap Y_{\alpha}$. Observe that each $Z_{\alpha}$ is clopen in $X$ being the inverse image of the clopen set $\oplus_{\beta<\alpha} Y_{\beta}$ intersected with the clopen set $Y_{\alpha}$. Put $Z=\oplus_{0<\alpha \leq \tau} Z_{\alpha}$. The triple $\left\{Z,\left.f\right|_{Z}, Y\right\}$ satisfies the hypothesis of Lemma 3.3. Therefore we can find a finite cover $\mathcal{F}_{Z}$ of $Z$ by colors such that $c l_{Y}(f(F)) \cap F=\emptyset$ for every $F \in \mathcal{F}_{Z}$.

For each isolated $\alpha \leq \tau$, put $P_{\alpha}=\left(X \cap Y_{\alpha}\right) \backslash Z_{\alpha}$. Since $Z_{\alpha}$ is open, $P_{\alpha}$ is closed in $Y_{\alpha}$. Since $f\left(Y_{\alpha}\right) \subset \bigcup_{\beta \leq \alpha} Y_{\beta}$ and $P_{\alpha}$ is outside of $Z_{\alpha}$, we conclude that $f\left(P_{\alpha}\right) \subset Y_{\alpha}$. For each isolated $\alpha \leq \tau$ the triple $\left\{Y_{\alpha}, P_{\alpha},\left.f\right|_{P_{\alpha}}\right\}$ meets the requirement of Proposition 3.1. Therefore, we can fix an $(n+3)$-sized family $\mathcal{F}_{\alpha}=\left\{F_{1}^{\alpha}, \ldots, F_{n+3}^{\alpha}\right\}$, which is a coloring for $\left.f\right|_{P_{\alpha}}$ and $c l_{Y}(f(F)) \cap F=\emptyset$ for every $F \in \mathcal{F}_{\alpha}$. Put $F_{i}=\oplus\left\{F_{i}^{\alpha}: \alpha \leq \tau\right\}$. Each $F_{i}$ is closed as the union of a discrete family of closed sets. Since $f\left(F_{i}^{\alpha}\right) \subset Y_{\alpha}$, we conclude that $c l_{Y}\left(f\left(F_{i}^{\alpha}\right)\right)$ misses $F_{i}^{\beta}$ if $\alpha \neq \beta$. By the choice of $\mathcal{F}_{\alpha}, c l_{Y}\left(f\left(F_{i}^{\alpha}\right)\right)$ misses $F_{i}^{\alpha}$. Therefore, $c l_{Y}\left(f\left(F_{i}\right)\right) \cap F_{i}=\emptyset$. Thus, $\mathcal{F}_{P}=\left\{F_{i}: i \leq n+3\right\}$ covers $\oplus_{\alpha} P_{\alpha}$ by colors and $c l_{Y}(f(F)) \cap F=\emptyset$ for every $F \in \mathcal{F}_{P}$. Thus, $\mathcal{F}_{Z} \cup \mathcal{F}_{P}$ is a desired coloring of $f$.

The existence of a special coloring in Theorem 3.4 implies that $\tilde{f}: \beta X \rightarrow$ $\beta Y$ is fixed-point free as well. Therefore, we have the following equivalence statement.

Theorem 3.5. Let $f: X \rightarrow Y$ be a continuous map defined on a closed subspace $X$ of a locally compact and paracompact space $Y$ of dimension $\operatorname{dim} Y \leq n$. Then $f$ is fixed-point free iff $\tilde{f}: \beta X \rightarrow \beta Y$ is fixed-point free.

We finish this section by giving an estimate for the chromatic number for maps as in the hypothesis of Theorem 3.4.

Theorem 3.6. Any continuous fixed-point free map $f: X \rightarrow Y$, defined on a closed subspace $X$ of a locally compact and paracompact space $Y$ of dimension $\operatorname{dim} Y \leq n$ is colorable in at most $(n+3)$ colors.

Proof. By Theorem 3.5, $\widetilde{f}$ has no fixed points. Consequently, by Proposition 3.1, $\tilde{f}$ is colorable in at most $n+3$ colors, which implies that the chromatic number of $f$ also does not exceed $n+3$. 


\section{Examples And Comments}

As mentioned in the Introduction Mazur constructed a continuous fixed-point free non-colorable map of the space of irrationals into itself. The space $P$ of irrational numbers is the first element in the hierarchy $\left\{N_{n}^{2 n+1}: n \in \omega\right\}$ of $n$ dimensional universal Nöbeling spaces (i.e. $P=N_{0}^{1}$ ).

Proposition 4.1. Let $n \in \omega$. There exists a continuous fixed-point free noncolorable self-map of the $n$-dimensional universal Nöbeling space $N_{n}^{2 n+1}$.

Proof. As noted, for $n=0$ this is known. Let $n>0$ and $f: N_{0}^{1} \rightarrow N_{0}^{1}$ be a continuous fixed-point free non-colorable map. Embed $N_{0}^{1}$ into $N_{n}^{2 n+1}$ as a closed subspace. By Lemma 2.8, since $\operatorname{dim} N_{n}^{2 n+1}=n$ and $N_{n}^{2 n+1} \in A E(n)$, we can embed $N_{0}^{1}$ into the product $N_{n}^{2 n+1} \times[0, \infty)$ as a closed subspace in such a way that $f=g \mid N_{0}^{1}$, where $g: N_{n}^{2 n+1} \times[0, \infty) \rightarrow N_{n}^{2 n+1} \times[0, \infty)$ is a fixed-point free map. Let $p: N_{n}^{2 n+1} \rightarrow N_{n}^{2 n+1} \times[0, \infty)$ be an $n$-soft map. Choose a section $i: N_{0}^{1} \rightarrow N_{n}^{2 n+1}$ of $p \mid p^{-1}\left(N_{0}^{1}\right)$, i.e. $p i=\operatorname{id}_{N_{0}^{1}}$, and let $A=i\left(N_{0}^{1}\right)$. Consider the map $j: A \rightarrow A$ defined by $j=i \circ f \circ p \mid A$ and note that $p \circ j=p \circ i \circ f \circ p \mid A=$ $f \circ p|A=g \circ p| A$. Since $p$ is $n$-soft there exists a map $r: N_{n}^{2 n+1} \rightarrow N_{n}^{2 n+1}$ such that $p \circ r=g \circ p$ and $r \mid A=j$. The former implies that $r$ is fixed-point free. The latter implies that $r$ is not colorable (otherwise $j$ would have been colorable; but since $j$ is equivalent to $f$ this is impossible).

Proposition 4.2. There exists a continuous fixed-point free non-colorable selfmap of the separable Hilbert space $\ell_{2} \simeq \mathbb{R}^{\omega}$.

Proof. Let $\mathbb{S}^{n}=\left\{x \in \mathbb{R}^{n+1}:\|x\|=\frac{1}{n+1}\right\} \times\left\{0_{i}\right\}_{i \geq n+2} \subset \mathbb{R}^{\omega}$. The map $f(\mathbf{x})=-\mathbf{x}$ is fixed-point free self-map of $\mathbb{P}=\mathbb{R}^{\omega} \backslash\{\mathbf{0}\}$ and coincides with the antipodal map on each $\mathbb{S}^{n}$. According to Van Douwen's observation $\widetilde{g}: \beta \mathbb{S} \rightarrow \beta \mathbb{S}$, where $\mathbb{S}=\cup \mathbb{S}^{n}$ and $g=f \mid \mathbb{S}$, fixes a point. Since $\mathbb{S}$ is closed in $\mathbb{P}$, it follows that $\tilde{f}$ fixes a point which means that $f$ is not colorable. Since $\ell_{2}$ is homeomorphic to $\mathbb{P}$ the needed conclusion follows.

Proposition 4.3. Let $f: X \rightarrow X$ be a continuous self-map of a finitedimensional locally compact paracompact space and $\widetilde{f}: \beta X \rightarrow \beta X$ be its extension. Then $\operatorname{Fix}(\tilde{f})=\operatorname{cl}_{\beta X} \operatorname{Fix}(f)$.

Proof. Obviously $\operatorname{cl}_{\beta X} \operatorname{Fix}(f) \subset \operatorname{Fix}(\widetilde{f})$. Suppose that there is $p \in \operatorname{Fix}(\widetilde{f}) \backslash$ $\mathrm{cl}_{\beta X} \operatorname{Fix}(f)$. Choose a functionally closed set $Z \subset X$ such that $p \in \operatorname{cl}_{\beta X} Z$ and $Z \cap \operatorname{Fix}(f)=\emptyset$. Let $\varphi: X \rightarrow[0, \infty)$ be a function such that $\varphi^{-1}(0)=Z$. Consider the map $g: X \times[0, \infty) \rightarrow X \times[0, \infty)$ defined by letting $g(x, r)=$ $(f(x), r+\varphi(x)),(x, r) \in X \times[0, \infty)$, and note that $g$ has no fixed points. The product $X \times[0, \infty)$ is still finite-dimensional, locally compact and paracompact. Therefore, by Theorem $3.5, \widetilde{g}: \beta(X \times[0, \infty)) \rightarrow \beta(X \times[0, \infty))$ has no fixed points. On the other hand, identifying $Z$ with $Z \times\{0\}$, we see that $f|Z=g| Z$ and consequently $\widetilde{g}(p)=\widetilde{f}(p)=p$. This contradiction completes the proof. 
The next observation shows that assumption of closedness of $X$ (in $Y$ ) in Theorem 3.4 (as well as in Propositions 2.9 and 3.1) cannot be dropped.

Proposition 4.4. There exists a continuous non-colorable fixed-point free map $g: X \rightarrow Y$, defined on an open subspace $X$ of a zero-dimensional metrizable compactum $Y$.

Proof. Let $f: P \rightarrow P$ be a continuous non-colorable fixed-point free self-map of the space $P$ of irrational numbers. Since the extension of $f$ onto the StoneCech compactification $\beta P$ of $P$ has fixed points, there exist a zero-dimensional metrizable compactification $Y$ of $P$ and a map $\tilde{f}: Y \rightarrow Y$ such that $\widetilde{f} \mid P=f$ and $\operatorname{Fix}(\widetilde{f}) \neq \emptyset$. Set $X=\widetilde{f}^{-1}(\operatorname{Fix}(\widetilde{f}))$ and $g=\widetilde{f} \mid X$.

The following statement can be considered as a converse (among Polish spaces) of [6, Theorem 3.3].

Proposition 4.5. Let $X$ be a zero-dimensional Polish space. Then the following conditions are equivalent:

(1) Every continuous fixed-point free self-map of $X$ is colorable;

(2) $X$ is sigma-compact.

Proof. $(1) \Longrightarrow(2)$. By $L C(X)$ denote the union of all open compact subsets of $X$. Let $X^{0}=L C(X), X^{\alpha+1}=X^{\alpha} \cup L C\left(X \backslash X^{\alpha}\right)$ and $X^{\alpha}=\cup\left\{X^{\beta}: \beta<\alpha\right\}$ for a limit ordinal. Note that $\left\{X^{\alpha}: \alpha<\omega_{1}\right\}$ is an increasing union of open subsets of $X$ and consequently $X^{\beta}=X^{\alpha}$ for some $\beta<\omega_{1}$ and all $\alpha>\beta$. Clearly $Y=X \backslash X^{\beta}$ has no points of local compactness. Note also that $X^{\beta}$ is sigma-compact. Assuming that $X$ is not sigma-compact, it follows that $Y \neq$ $\emptyset$. Since $Y$ is completely metrizable (as a closed subspace of $X$ ) and zerodimensional, we conclude that $Y$ is homeomorphic to the space of irrational numbers. Let $g: Y \rightarrow Y$ be a continuous non-colorable fixed-point free map (Mazur's example) and $r: X \rightarrow Y$ be a retraction. Then the composition $f=g \circ r: X \rightarrow X$ is a non-colorable fixed-point free map contradicting (1).

$(2) \Longrightarrow(1)$. This follows from [6].

Our main theorem for locally compact Lindelöf spaces of finite dimension and the Krawczyk-Steprāns result for zero-dimensional $\sigma$-compact spaces [6, Theorem 3.3] motivate the following question.

Question 4.6. Let $X$ be a $\sigma$-compact space of finite dimension. Is every continuous fixed-point free self-map on $X$ colorable? 


\section{REFERENCES}

[1] R. Z. Buzyakova, Fixed-point free maps of the reals and more, Topology Appl. 156 (2008), 465-472.

[2] A. Chigogidze, Inverse Spectra, North Holland, Amsterdam, 1996.

[3] E. van Douwen, $\beta X$ and fixed-point free maps, Topology Appl. 51 (1993), 191-195.

[4] R. Engelking, General Topology, PWN, Warszawa, 1977.

[5] M. Katětov, A theorem on mappings, Comm. Math. Univ. Carolinae 8 (1967), 431-433.

[6] A. Krawczyk, J. Steprāns, Continuous colourings of closed graphs, Topology Appl. 51 (1993), 13-26.

[7] J. van Mill, Easier proofs of coloring theorems, Topology Appl. 97 (1999), 155-163.

[8] J. van Mill, The infinite-dimensional topology of function spaces, Elsevier, Amsterdam, 2001.

Department of Mathematics and Statistics, The University of North Carolina at Greensboro, Greensboro, NC, 27402, USA

E-mail address: rzbouzia@uncg.edu

Department of Mathematics and Statistics, The University of North Carolina at Greensboro, Greensboro, NC, 27402, USA

E-mail address: chigogidze@uncg.edu 